\title{
Quality Spectrum of RNA in Cryopreserved Cancer Biospecimens
}

\section{Background:}

In the era of personalized medicine, the availability of biospecimen is a valuable and indispensable resource for translational research. Only getting the biospecimen is not a solution but getting a good quality and well preserved one is the key to obtain reliable and reproducible results. The cryo preserved biospecimens can thus be used for genomics or proteomics study. DNA and proteins remain stable for years but RNA being prone to degradation has a shorter shelf life, the quality of cryopteserved specimens are better estimated by RNA integrity. To address the issue of long term cryopreservation we analyzed the quality of RNA in this study.

\section{Methods:}

$>$ Inclusion Criteria

1. Specific tumor type

2. $>70 \%$ viable tumor excluding necrosis,

inflammation, stromal cells, epithelial cells and fibrosis.

$>$ Screened : 92 Tissues

$>$ Included : 34 samples and RNA was extracted

(Agilent RNA isolation mini kit). RNA integrity

denaturing gel electrophoresis (MOPS) for intact

$28 \mathrm{~S}$ and $18 \mathrm{~S}$ bands.

$>$ RNA quantification : Nanodrop and quality of samples was verified by A260/280 and A260/230 ratio \& Bioanalyzer.

$>$ Quality of cDNA : Real time PCR (q-PCR) through amplification of housekeeping genes (RPS13, GAPDH and $\beta$-Actin).

\section{Results:}

$>$ The period of cryopreservation of these biospecimens varies from 6-30 months. RNA integrity was maintained for $>85 \%$ of the cryopreserved biospecimens.

$>$ The quantity varies from $500-1950$ $\mathrm{ng} / \mu \mathrm{L}$.

$>$ Mean A260/280 \& A260/230 ratio for breast, brain, testis and stomach samples were $2.02,2.03,2.02,2.07$ and 1.32 ,

$1.54,1.26,1.76$ respectively.

$>$ The Spearman correlation (r) analysis confirmed association of RIN and A260/280 ratio with $p<0.01$ and 95\% confidence interval (two-tailed test).

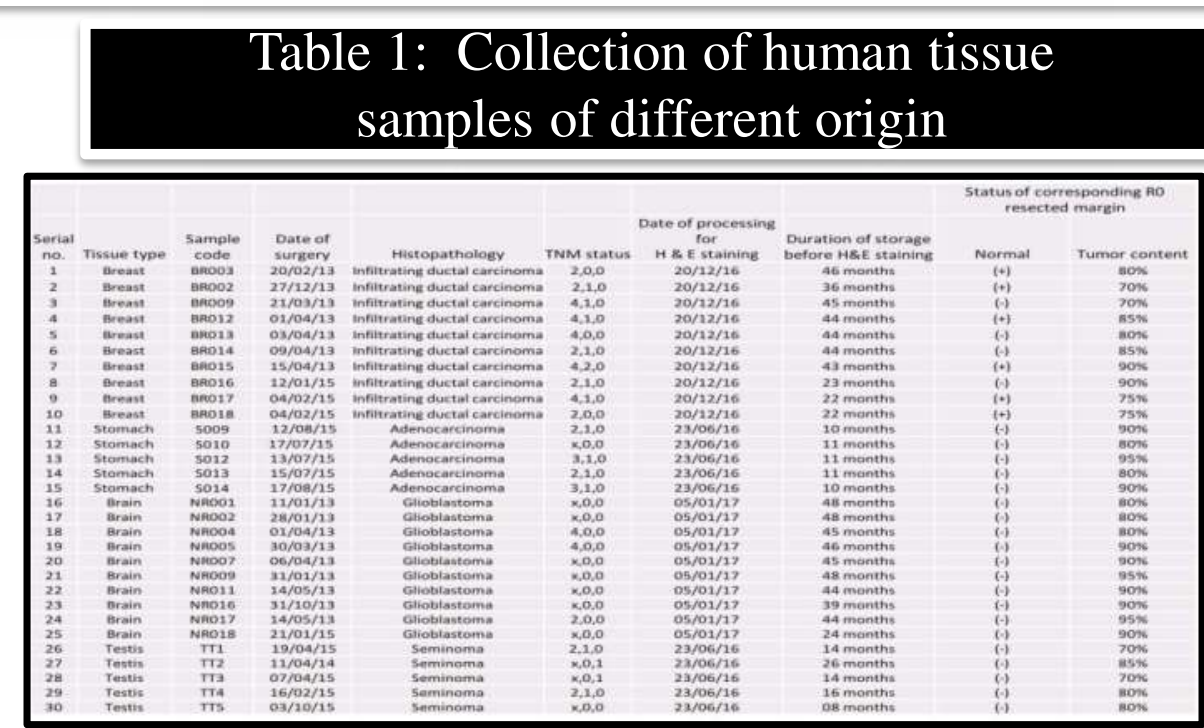

Table 2: RNA yield and quality of RNA from human tissue samples

Fig 2: Correlation of RIN and A260/280 ratio of human tissue samples

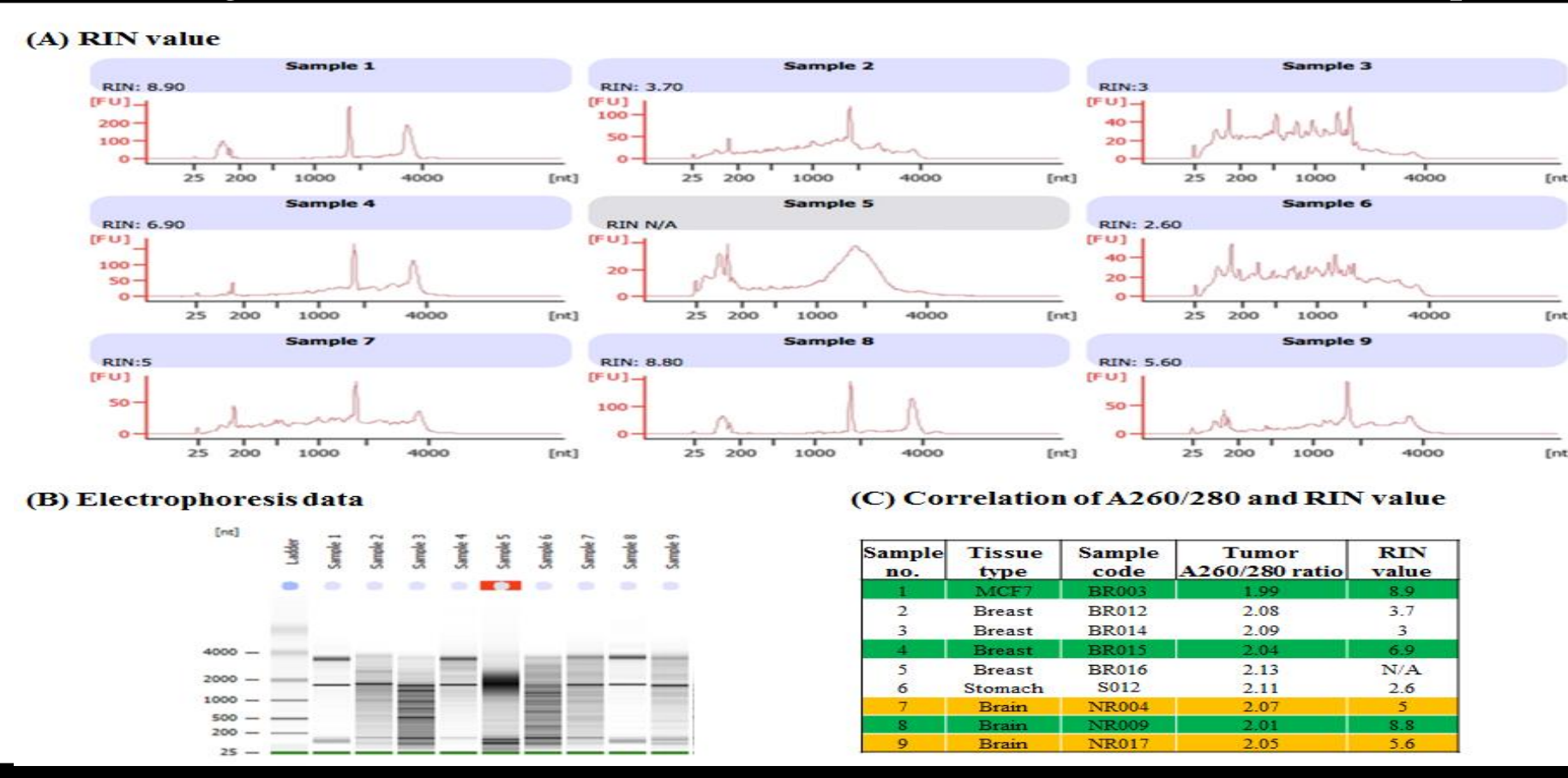

Fig 3: cDNA quality assessment by real time PCR of human tissue sample
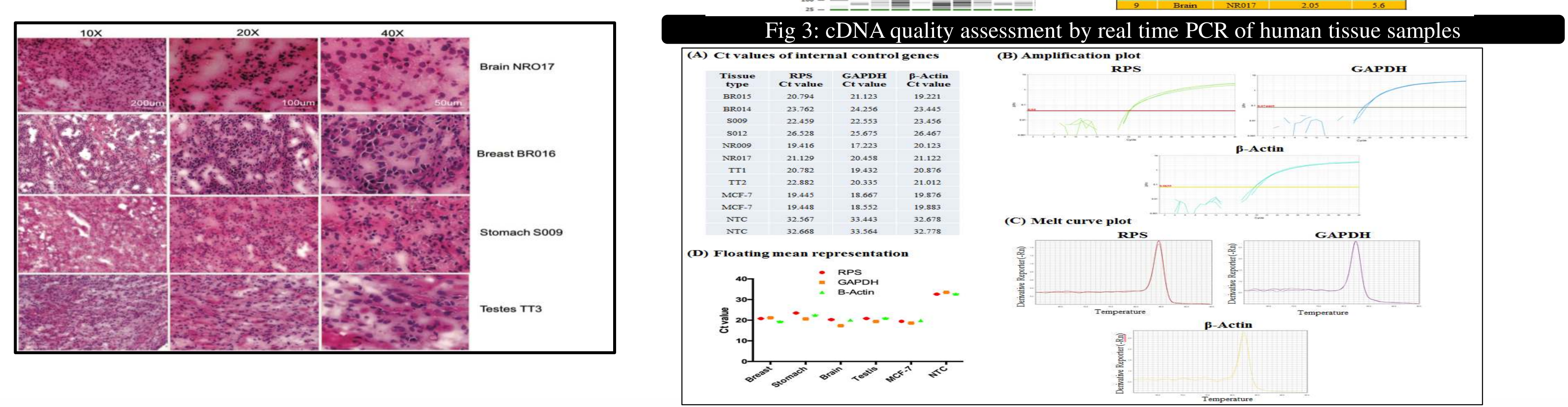

\section{Conclusion:}

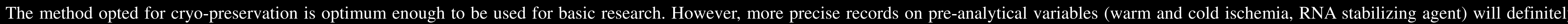
enhance the knowledge affecting quality of RNA.

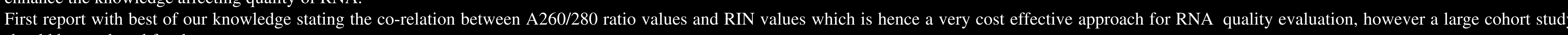
should be analyzed for the same. 